\title{
Analysis of 802.11 OFDM in High Multipath Environments
}

\author{
F. Heereman ${ }^{1}$, W. Joseph ${ }^{1}$, L. Martens ${ }^{1}$ \\ ${ }^{1}$ Ghent University/iMinds, Department of Information Technology, \\ Gaston Crommenlaan 8 box 201, B-9050 Ghent, Belgium, e-mail*: frederic.heereman@intec.ugent.be
}

\begin{abstract}
The performance loss of 802.11 OFDM systems due to propagation delay spread has been analyzed as a function of OFDM parameters for a wide range of reverberation times. This analysis results into solutions for the OFDM design to suppress the performance degradation.
\end{abstract}

Index Terms-delay spread; diffuse multipath; reverberation time; OFDM; room electromagnetics

\section{INTRODUCTION}

The performance of OFDM (orthogonal frequency-division multiplexing) systems can be degraded by the signal distortion over the FFT (fast Fourier transform) window caused by the propagation delay spread. In [1], we proposed to describe this effect in narrowband OFDM systems (such as IEEE $802.11 \mathrm{a} / \mathrm{g} / \mathrm{h} / \mathrm{ac}$ ) by an additive white Gaussian noise (AWGN) noise, characterized by a noise factor $F_{\text {delay }}$. This effect originates from replicas of the transmitted OFDM pulse with a delay larger than the cyclic prefix length $C P$. The intensity of these propagation paths can be high, especially in indoor environments, resulting into intersymbol and intercarrier (ISI/ICI) interference. For delays higher than $C P$, the channel typically consists of diffuse multipath components only. Here, the theory of room electromagnetics is applicable [2], according to which the averaged power delay profile (APDP) decays exponentially. This APDP decay is characterized by the time constant $\tau_{r}$, referred to as the reverberation time, and the intensity parameter $I_{\text {diff }}[\mathrm{Hz}][1]$. As $P_{0}$, the APDP power coefficient of first arriving path, is dependent on the frequency width $\Delta f_{0}$ of the Hann window applied to obtain the APDP, the intensity of the diffuse field will be expressed by the physical parameter $I_{d i f f}$, defined by $I_{d i f f}=P_{0} \Delta f_{0}$ [1]. Based on this theory, an analytical expression of $F_{\text {delay }}$ has been developed in [1] in terms of OFDM parameters and the propagation parameters $\tau_{r}$ and $I_{d i f f}$.

In this work, a parametric analysis of $F_{\text {delay }}$ is carried out as a function of OFDM parameters, based on the aforementioned analytical expression for $F_{\text {delay. }}$. This analysis is done for typical IEEE $802.11 \mathrm{a} / \mathrm{g} / \mathrm{n} / \mathrm{ac}$ parameters [3] [4]. This gives insight and solutions for the OFDM design to suppress the performance loss due to the propagation delay spread.

\section{Determination of $F_{\text {delay }}$}

In [1], the performance loss due to the signal distortion over the FFT window (caused by the propagation delay spread), described by a loss factor $L_{\text {delay }}$, has been related to the noise factor $F_{\text {delay }}$ as follows:

$$
L_{\text {delay }}=1+\frac{F_{\text {delay }}}{F_{\text {lin }} I L_{\text {lin }}},
$$

where $F_{l i n}$ and $I L_{l i n}$ are the conventional (linear-scaled) noise factor and implementation loss of the receiver, resp. (i.e., corresponding to the situation where receiver and transmitter are connected by a cable). Therefore, our analysis will be done in terms of $F_{\text {delay }}$.

For the purpose of this work, we rewrite the expression for $F_{\text {delay }}$ from [1] as a function of the following relevant OFDM design parameters: the transmit power per frequency unit $P_{T, f}$, the total bandwidth $B W$ of the channel, the FFT period $P, C P$ and the sampling factor $f_{s}$. The number of samples per FFT period $\left(N_{\text {sample }}\right)$ is typically higher or equal than the total number of subcarriers, being $B W \times P$. $N_{\text {sample }}$ is usually expressed by means of the sampling factor $f_{s}$ :

$$
N_{\text {sample }}=f_{s} B W P .
$$

\section{PARAMETRIC ANALYSIS}

In this section, the analytical estimation for $F_{\text {delay }}$ is analyzed as a function of $P, C P, B W$ and $f_{s}$. All calculations of $F_{\text {delay }}$ presented in this work are, unless otherwise mentioned, based on the 802.11a physical standard: $P=3.2 \mu \mathrm{s}$, $C P=800 \mathrm{~ns}, B W=20 \mathrm{MHz}$ and $f_{s}=1$. We assume a typical value for $I_{\text {diff } f}$ of $6 \mathrm{~Hz}$ and a wide range of $\tau_{r}$ varying from $10 \mathrm{~ns}$ to $200 \mathrm{~ns}$, based on experimental results [1]. For our calculations, we assume $P_{T, f}=6.210^{-9} \mathrm{~W} / \mathrm{Hz}$, based on a $20 \mathrm{dBm}$ transmit power. For a $30 \mathrm{dBm}$ transmit power, $F_{\text {delay }}$ can be simply found as $10 \mathrm{~dB}$ higher, as $F_{\text {delay }}$ is proportional to the transmit power [1].

\section{A. Influence of the cyclic prefix duration $C P$}

In Fig. $1, F_{\text {delay }}$ is shown as a function of $C P$ for different $\tau_{r} . F_{\text {delay }}$ decreases strongly with increasing $C P$, due to the fact that $F_{\text {delay }}$ is proportional to $\exp \left(-C P / \tau_{r}\right)$. Although the dependency of $F_{\text {delay }}$ on $C P$ is less strong for higher $\tau_{r}$, increasing $C P$ still provides an efficient strategy to reduce the interference due to delay spread. E.g., for $\tau_{r}=140 \mathrm{ns,}$ $F_{\text {delay }}$ decreases from $28.6 \mathrm{~dB}$ to $3.8 \mathrm{~dB}$ when switching from an $800 \mathrm{~ns} C P$ to $1600 \mathrm{~ns}$. This corresponds to a loss $L_{\text {delay }}$ reduction of about $14 \mathrm{~dB}$ (see (1)). For a $30 \mathrm{dBm}$ transmit power, the decrease of $F_{\text {delay }}$ is even from $38.6 \mathrm{~dB}$ to $13.8 \mathrm{~dB}$. When switching from an $800 \mathrm{~ns} C P$ to $1600 \mathrm{~ns}$, the 
data rate is reduced with about $17 \%$. However, this is largely compensated by the strong reduction of $L_{\text {delay }}$.

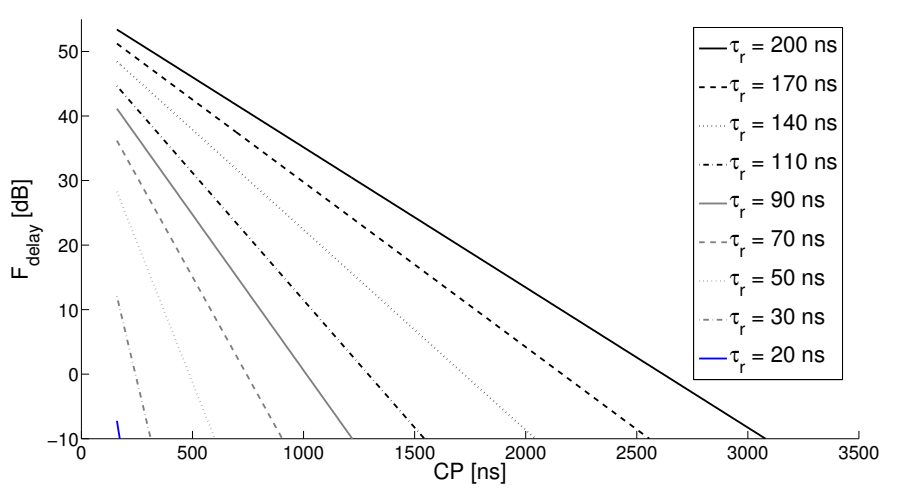

Figure 1. Calculated noise factor $F_{\text {delay }}$ as a function of cyclic prefix $C P$ for different reverberation time $\tau_{r}$. This is based on IEEE 802.11a and a $20 \mathrm{dBm}$ transmit power.

\section{B. Influence of the FFT period $P$}

We found that $F_{\text {delay }}$ is inversely proportionally to $P$. In our analysis, the effect on the theoretical (transmission) data rate $R_{\text {data }}$ (proportional to $P /(P+C P)$ ) and the hardware complexity (related to the number of used subcarriers, proportional to $B W \times P$ ) is taken into account simultaneously. A higher FFT period $P$ would result in a lower performance loss due to delay spread $\left(F_{\text {delay }}\right)$ as well as higher data rate, but the FFT processor would also require a higher size. When switching from $P=3.2 \mu$ s to e.g., $6.4 \mu \mathrm{s}, F_{\text {delay }}$ would decrease with $3 \mathrm{~dB}$ and the data rate would increase with $11 \%$. However, the FFT size would increase from 64 to 128 . Therefore, increasing $P$ is not really an efficient strategy to suppress the performance loss due to delay spread.

\section{Influence of the bandwidth $B W$}

Increasing the bandwidth results into an increased $F_{\text {delay }}$ due to a reduced sampling period, which acts as an extension of the cyclic prefix. The dependency of $F_{\text {delay }}$ on $B W$ is rather slight. Comparing $B W=160 \mathrm{MHz}(802.11 \mathrm{ac})$ to $20 \mathrm{MHz}$, the increase of $F_{\text {delay }}$ is only $3 \mathrm{~dB}$ for $\tau_{r}=50 \mathrm{~ns}$ and $2 \mathrm{~dB}$ for $\tau_{r}=70 \mathrm{~ns}$.

\section{Influence of the sampling factor $f_{s}$}

$F_{\text {delay }}$ increases slightly for increasing $f_{s}$. E.g., when changing $f_{s}$ from 1 to 4 , there is an increase of $F_{\text {delay }}$ by $0.6 \mathrm{~dB}$ for $\tau_{r}=200 \mathrm{~ns}$ and $2.5 \mathrm{~dB}$ for $\tau_{r}=50 \mathrm{~ns}$.

\section{IMPLICATIONS TO OFDM DESIGN}

Our analysis shows that, to suppress the noise factor $F_{\text {delay }}$ due to delay spread, an efficient strategy is related to the increase of the cyclic prefix length $C P$ (i.e., guard interval (GI)). When switching to a long GI option of $1600 \mathrm{~ns}$ (from $800 \mathrm{~ns}$ GI), $F_{\text {delay }}$ is reduced by even $17.4 \mathrm{~dB}$ for $\tau_{r}=200 \mathrm{~ns}$, and by $24.8 \mathrm{~dB}$ for $\tau_{r}=140 \mathrm{~ns}$. The data rate $R_{\text {data }}$ is reduced by
$17 \%$, but this is largely compensated by the strong reduction of $F_{\text {delay }}$.

The strategy of an increased $C P$ is easy with respect to the implementation, but the theoretical data rate $R_{\text {data }}$ is reduced. To keep this data rate constant, the ratio between $P$ and $C P$ should be kept constant. As mentioned before, this requires a higher hardware complexity. However, in systems with a higher bandwidth mode, such as $802.11 \mathrm{n}(40 \mathrm{MHz})$ and 802.11ac (40/80/160 MHz), the more complex hardware could be combined with the principle of scaled OFDM [5]. This would provide a method for systems with a higher bandwidth mode to implement a long GI option for a lower bandwidth mode, without reduction of the (theoretical) data rate and without requiring a complex hardware extension.

\section{CONCLUSION}

In this work, the performance loss due to delay spread (in terms of $F_{\text {delay }}$ ) has been analyzed as a function of OFDM parameters for a wide range of the reverberation time (i.e., $10-200 \mathrm{~ns})$. This loss, caused by diffuse multipath, can be severe: e.g., $F_{\text {delay }}=38.6 \mathrm{~dB}$ for $C P=800 \mathrm{~ns}$, a $30 \mathrm{dBm}$ transmit power and a high (but realistic) $\tau_{r}=140$ ns. $F_{\text {delay }}$ decreases exponentially with increasing $C P$. E.g., for $\tau_{r}=$ $140 \mathrm{~ns}$, there is a reduction of $F_{\text {delay }}$ by about $25 \mathrm{~dB}$, when switching $C P$ from $800 \mathrm{~ns}$ to $1600 \mathrm{~ns}$. Further, we found that $F_{\text {delay }}$ decreases inversely proportionally with increasing $P$. Taking into account the implications on the theoretical data rate and the hardware complexity, we propose to adopt a long guard interval option to the 802.11 OFDM standard to ensure reliable reception in high multipath environments. In future research, the analysis presented will be validated experimentally.

\section{REFERENCES}

[1] F. Heereman, W. Joseph, E. Tanghe, L. Verloock, and L. Martens, "Performance degradation due to multipath noise for narrowband OFDM systems: Channel-based analysis and experimental determination," IEEE Trans. Wireless Commun. (submitted).

[2] J. Andersen, J. Nielsen, G. Pedersen, G. Bauch, and M. Herdin, "Room electromagnetics," IEEE Antennas and Propagation Magazine, vol. 49, no. 2, pp. 27-33, 2007.

[3] IEEE Std 802.11n $n^{T M}-2009$ amendment 5 to part 11: Wireless LAN Medium Access Control (MAC) and Physical Layer (PHY) specifications: enhancements for higher throughput, IEEE Std., Oct. 2009.

[4] "802.11ac technology introduction," Rohde \& Schwarz, Tech. Rep., Mar. 2012.

[5] IEEE Std part 11: Wireless LAN Medium Access Control (MAC) and Physical Layer (PHY) specifications: Amendment 3: $3650-3700 \mathrm{MHz}$ Operation in USA, IEEE Std., Nov. 2008. 